\title{
Pathological Diagnosis and Genomic Characterization of ICP4 Gene of lnfectious Laryngotracheitis Virus (ILTV) Isolates in Clinically Infected Chicken in Tamil Nadu, India
}

\author{
R. Jyothi Priya, Ganne Venkata Sudhakar Rao, N. Pazhanivel,
}

K. Vijayarani, T. Lurthu Reetha, V. Gowthaman, P. Raja

10.18805/IJAR.B-4802

\begin{abstract}
Background: Infectious laryngotracheitis (ILT) is an economically important viral respiratory disease in poultry. Recently, re-emergence of Infectious laryngotracheitis virus (ILTV) has been reported in several countries including India. The current study aimed to evaluate the poultry flocks of Tamil Nadu with circulating GaHV-1 and to elucidate the origin of the virus involved in the outbreak.

Methods: In this study, a molecular based survey on the overall occurrence of natural cases of Infectious laryngo-tracheitis in poultry flocks from Tamil Nadu, India were performed. Pathological findings in respiratory and secondary lymphoid organs like caecal tonsils and harderian gland was carried out. The PCR technique targeting Infected Cell Protein-4 (ICP4) gene along with molecular characterization was performed.

Result: The overall prevalence rate in the outbreak was $42.86 \%$ with highest incidence in layer flocks $(62.85 \%)$ than the broiler flocks $(22.85 \%)$. The highest susceptible age groups were between 20-30 weeks old. Tracheal pathology revealed epithelial sloughing, syncytial cell formation, eosinophilic intranuclear inclusion bodies and heterophilic exudation microscopically. Partial genome sequencing and Phylogenetic analysis of ICP4 gene revealed high genetic homology between field isolates and the virulent strains from Turkey, Germany, China and Brazil. In the present study, along with pathological findings, a rapid and sensitive PCR assay was used for detection of ILT virus specific ICP4 gene in commercial poultry farms in the region.
\end{abstract}

Key words: Genomic characterization, ICP4 gene, Infectious laryngotracheitis (ILT), Pathology, PCR, Phylogenetic analysis.

\section{INTRODUCTION}

Infectious laryngotracheitis (ILT) is classified as a disease requiring mandatory notification to the World Organization for Animal Health (OIE, 2021). It is an acute, highly contagious upper-respiratory infectious disease of chickens, which was first described in the USA in 1925 (May and Tittsler, 1925). It is one among the emerging and re-emerging diseases of poultry gradually spreading worldwide and affects chickens of all age groups (Menendez et al., 2014). This disease causes severe economic losses to poultry industry due to decrease in egg production, weight loss, susceptibility to other respiratory infections, high mortality and morbidity (Guy and Bagust, 2003). According to the Ninth International Committee on the Taxonomy of Viruses (ICTV), Gallid herpesvirus 1(GaHV 1), is also called as Infectious laryngotracheitis virus (ILTV) which is a doublestranded DNA virus belonging to family Herpesviridae, subfamily Alphaherpesvirinae, genus Iltovirus (King et al., 2012). The primary natural hosts of ILTV are chickens and the disease is commonly observed in layer hens. Backyard poultry flocks also act as an important source of infection for commercial poultry flocks and have recently been reported at higher frequencies (Moreno et al., 2010). In chickens, two main forms of ILT includes the severe acute form characterized by significant respiratory distress, expectoration of blood mixed mucus and severe
Department of Veterinary Pathology, Madras Veterinary College, Tamil Nadu Veterinary and Animal Sciences University, Chennai600 051, Tamil Nadu, India.

Corresponding Author: R. Jyothi Priya, Department of Veterinary Pathology, Madras Veterinary College, Tamil Nadu Veterinary and Animal Sciences University, Chennai-600 051, Tamil Nadu, India. Email: drjpriya@rediffmail.com

How to cite this article: Priya, R.J., Rao, G.V.S., Pazhanivel, N., Vijayarani, K., Reetha, T.L., Gowthaman, V. and Raja, P. (2022). Pathological Diagnosis and Genomic Characterization of ICP4 gene of Infectious Laryngotracheitis Virus (ILTV) Isolates in Clinically Infected Chicken in Tamil Nadu, India. Indian Journal of Animal Research. DOI: 10.18805/IJAR.B-4802.

Submitted: 09-10-2021 Accepted: 22-11-2021 Online: 11-01-2022

haemorrhagic tracheitis accompanied by high mortality ranging from 5 to $70 \%$ and a milder form characterized by mild to moderate catarrhal tracheitis, sinusitis, conjunctivitis, relatively low morbidity and occasional mortality which usually range between 0.1 and $2 \%$ (Ou and Giambrone, 2012).

DNA detection by conventional polymerase chain reaction $(P C R)$ has become a preferred method of virus diagnosis (OIE, 2021) due to its improved rapidity, sensitivity and reproducibility. DNA sequencing can be used for monitoring chickens and through corresponding 
epidemiological studies, the origins of the viruses circulating and involved in outbreaks can be identified in addition to identifying possible recombination events (Lee et al., 2013). Sequencing of specific gene fragments including the Infected Cell Polypeptide-4 (ICP4) gene is commonly used to determine the genotypic origin of the ILT strains in field infections and it seems to be an efficient tool to characterize field isolates and differentiate them from vaccine strains (Chacon and Ferreira, 2009).

Recently, the disease has gained whim as evidenced by many reports on severe economic losses due to ILT outbreak in India (Bhutia and Singh, 2017) and the state of Tamil Nadu during the last six years (Gowthaman et al., 2014; Sivaseelan et al., 2014; Mishra et al., 2020) which is known for its commercial poultry production hub in India. Hence, the objective of this study was to evaluate poultry flocks of the region with circulating GaHV-1 and to elucidate the origin of the virus involved in the outbreak for a period of two years (2019-2021), using histopathology and molecular characterization of the virus, coupled with phylogenetic reconstructions based on sequences of ICP4 gene.

\section{MATERIALS AND METHODS}

\section{Sampling and tissue processing}

The disease investigation was carried out in 35 commercial poultry layer flocks ( $n=210$ birds) and 35 broiler flocks $(n=210$ birds) of Tamil Nadu including districts covered under cauvery delta zone and western agro-climatic zones of the state. The flocks investigated were presented with a history of generalized depression, respiratory signs like conjunctivitis, sinusitis, gasping, oculo-nasal discharge and reduced egg production during the period between September, 2019 and August, 2021. A random of six moribund/dead birds per flock was brought to the laboratory and necropsy examination was carried out. Oro-pharyngeal swabs and tissue samples like trachea, lungs, harderian gland and caecal tonsil were collected aseptically for the virus detection and disease diagnosis. Heart blood swabs, tracheal swabs and tracheas were collected from dead birds for bacterial and viral examination. Heart blood swabs were inoculated into blood agar, brain heart infusion agar, nutrient agar and MacConkey agar plates.

\section{Gross pathology and histopathology}

A detailed necropsy examination was carried out. Following necropsy, organs like trachea, lungs, caecal tonsils and harderian gland were examined grossly and fixed in $10 \%$ neutral buffered formalin for histopathology processing (Bancroft and Gamble, 2008) and subsequent microscopy.

\section{Total DNA extraction and polymerase chain reaction}

DNA extraction from the samples was done by DNeasy Blood and Tissue Kit (M/s Qiagen, India) as specified by the manufacturer's instructions. The obtained DNA samples were stored at $-20^{\circ} \mathrm{C}$. Molecular detection of ILTV genome was carried out by conventional PCR amplifying ICP4 gene of ILTV (Chacon and Ferreira, 2009; OIE, 2021). PCR analysis was performed using the primers ICP4-1F and ICP4-1R (Table 1). The extracted DNA was amplified in a total volume of $25 \mu \mathrm{L}$ (Table 2) using $2 X$ EmeraldAmp GT PCR Master mix (M/s. Takara, USA). The PCR cycling conditions with the Biorad gradient (T100) were given in Table 3 . The PCR products $(10 \mu \mathrm{L})$ were analysed by electrophoresis in $1.5 \%$ agarose gel and the gel was stained with ethidium bromide $(1.5 \mathrm{~g} / \mathrm{mL})$ and photographed. For positive control, DNA extracted from a lyophilized ILTV vaccine was used. The negative control contained only PCR master mix and primers.

\section{Nucleotide sequencing and blast analysis}

Bulk PCR products were purified using Mini Elute Gel extraction kit (Qiagen, USA) as per the manufacturer's instructions. For strain identification, the eluted PCR products from the field isolates were sent for Sanger dideoxy sequencing to $\mathrm{M} / \mathrm{s}$ Eurofins, Bangalore. The nucleotide sequences obtained were aligned with the known reference sequences of ILTV published in GenBank by using NCBI BLAST and Megalign software and were subjected to their homology scores alignment (Hall, 1999).

\section{Phylogenetic analysis of partial ICP4 gene}

The representative aligned sequences were submitted to GenBank for Accession number. Phylogenetic analysis was carried out with MEGA(X) ${ }^{\mathrm{TM}}$ software using Tamura-Nei model, maximum likelihood analyses with 1000 bootstrap values (Tamura and Nei,1993; Kumar et al., 2018).

\section{RESULTS AND DISCUSSION \\ ILTV screening among flocks}

In the present investigation, a total of 35 broiler and 35 layer farms were screened and tested for the presence of ILTV by the conventional PCR test. The overall prevalence of ILTV among poultry farms was found to be $42.86 \%$ (30/70) (Table 4). Among the selected type of flocks, the highest prevalence of $62.85 \%(22 / 35)$ was found in layer flock and the lowest prevalence of $22.85 \%(08 / 35)$ was observed in broiler flocks including native chicken. The morbidity rate was $40-65 \%$ while the daily mortality ranged from 1.83 to $16.67 \%$ with average mortality of $7.42 \%$ was recorded. This

Table 1: Oligonucleotide primers used for amplification of ICP4 gene of suspected ILTV infected field cases (Chacón and Ferreira, 2009; OIE, 2021).

\begin{tabular}{lllc}
\hline Target gene & Primers & Oligonucleotide sequence & Length of amplified fragment \\
\hline ICP4 Gene & ICP4-2F & $5^{\prime}$-CTTCAGACTCCAGCTCATCTG-3' & 635 bp \\
& ICP4-2R & 5'-AGTCATGCGTCTATGGCGTTGAC-3' & \\
\hline
\end{tabular}


Pathological Diagnosis and Genomic Characterization of ICP4 Gene of lnfectious Laryngotracheitis Virus (ILTV) Isolates in...

severe form of ILT outbreak correlated with earlier reports elucidating that severe form of ILT, the morbidity and mortality ranges from $90-100 \%$ and $5-70 \%$ respectively (Bhutia and Singh, 2017). Whereas, in milder form of ILTV infection, the morbidity is low up to $5 \%$ and mortality ranges from 0.5 to $2 \%$. No bacteria of pathogenic importance could be isolated from the tracheal swabs and heart blood swabs in the current outbreak indicating the absence of bacterial organisms.

\section{Clinical signs}

In this study, the clinical course of ILT varied from 5 days to 5 weeks and the birds revealed per acute, acute and chronic form of disease. The morbidity rate in the affected flocks was from $40 \%$ to $100 \%$ and the mortality ranged from $10 \%$ to $40 \%$ lasting up to $2-3$ weeks of the disease course. The affected flocks revealed sudden increase in average daily mortality. The egg production in severely affected flocks reduced from $30 \%$ up to $70 \%$. The affected birds became lethargic with head shaking, gasping and squinted eyes. Laboured breathing and expectoration of blood-stained mucus (Fig 1a) were observed in severely affected cases. In some farms, conjunctivitis, swelling of the infraorbital sinuses and closed eyes were noticed. Earlier studies have also reported varied range of clinical signs like rapid and high mortality rate exceeding 50\% (Preis et al., 2013), Coughing and gurgling with birds ejecting the clotted blood orally (Blakey et al., 2019) and varying level of decreased

Table 2: PCR reaction mixture.

\begin{tabular}{lc}
\hline Reactants (ICP4 gene) & Volume \\
\hline PCR master mix & $12.5 \mu \mathrm{L}$ \\
ICP4-2F (Forward primer) & $1.0 \mu \mathrm{L}$ \\
ICP4-2R (Reverse primer) & $1.0 \mu \mathrm{L}$ \\
DNA template & $3.0 \mu \mathrm{L}$ \\
Nuclease free water & $7.5 \mu \mathrm{L}$ \\
Total volume & $25.0 \mu \mathrm{L}$ \\
\hline
\end{tabular}

Table 3: PCR cycle condition.

\begin{tabular}{llcc}
\hline SI.No. & Steps & $\begin{array}{c}\text { Temperature } \\
\left({ }^{\circ} \mathrm{C}\right)\end{array}$ & Time \\
\hline Step 1 & Initial denaturation & 94 & $3 \mathrm{~min}$ \\
Step 2 & Denaturation & 94 & $1 \mathrm{~min}$ \\
Step 3 & Annealing & 62 & $30 \mathrm{sec}$ \\
Step 4 & Extension & 72 & $90 \mathrm{sec}$ \\
Step 5 & Step 2-4 were repeated for 35 cycles & & \\
Step 6 & Final extension & 72 & $7 \mathrm{~min}$ \\
Step 7 & Holding temperature & 4 & Hold \\
\hline
\end{tabular}

egg production in the affected flocks (Ou and Giambrone, 2012).

\section{Gross pathology}

In the per-acute cases and severe form of the disease, haemorrhagic tracheitis with blood clots (Fig 1b) and occlusion of tracheal and laryngeal lumen by whitish or yellowish or reddish plaques were observed. Pneumonic changes of congestion and red hepatisation were noticed in the lungs of the severely affected birds. Mild congestion was observed in caecal tonsils in the affected birds. In the acute form of the disease, yellow caseous diphtheritic membranes adherent to the larynx and proximal tracheal mucosa were observed. In sub-acute cases and less severe form of the disease, the laryngeal and tracheal mucosa showed discrete congestion and generalised catarrhal changes. The above findings correlated with the previous reports (Guy and Bagust, 2003).

\section{Histopathology}

Microscopically, trachea from the per acute stage of the disease showed congestion of blood vessels, disruption of the intact mucosa, hemmorrhagic exudate (Fig 1c) with lymphohistiocytic infiltrations in the mucosal and submucosal layer of the trachea, epithelial sloughing and deciliation. Formation of multinucleated epithelial cells (syncytia) with the presence of eosinophilic intranuclear inclusions (Fig 1d) was observed in the acute stage of the disease. During the sub-acute stage, the tracheal lumen revealed varied exudates containing fibrin, inflammatory cells, red blood cells, sloughed epithelial cells and syncytial cells with or without intranuclear inclusion bodies. Lung from the affected birds showed haemorrhages in the parabronchus (Fig 1e); the primary and secondary bronchi were characterized by epithelial degeneration and denudation with infiltration of heterophilic cells (Fig 1f). The secondary lymphoid organs such as harderian gland (Fig 1g) and caecal tonsils (Fig $1 \mathrm{~h}$ ) revealed mild lymphoid cell depletion and few necrotic foci in the lymphoid nodules. These findings were well correlated with the earlier studies (Bagust et al., 2000) and the reports from the same geographical location (Mishra et al., 2020). The Harderian gland in birds are considered as a peripheral lymphoepithelial organ (secondary lymphoid organ) which, together with spleen, bursa of fabricius and caecal tonsils resolute both general and local immunity and are a source of immune response in birds (Mobini, 2012). Hence, the mild lymphocyte depletion and necrotic foci in the harderian gland and caecal tonsil in the affected birds elicited that the ILTV is an immunosuppressive pathogen.

Table 4: Prevalence of ILTV among the type of poultry flocks.

\begin{tabular}{lcccc}
\hline Farm type & $\begin{array}{c}\text { Total no. of farms } \\
\text { investigated }\end{array}$ & $\begin{array}{c}\text { No. of birds screened } \\
(\mathrm{n}=6)\end{array}$ & $\begin{array}{c}\text { Farms positive } \\
\text { for ILTV }\end{array}$ & $\begin{array}{c}\text { Per cent prevalence } \\
(\%)\end{array}$ \\
\hline Broilers & 35 & 210 & 08 & 22.85 \\
Layers & 35 & 210 & 22 & 62.85 \\
Total & 70 & 420 & 30 & 42.86 \\
\hline
\end{tabular}




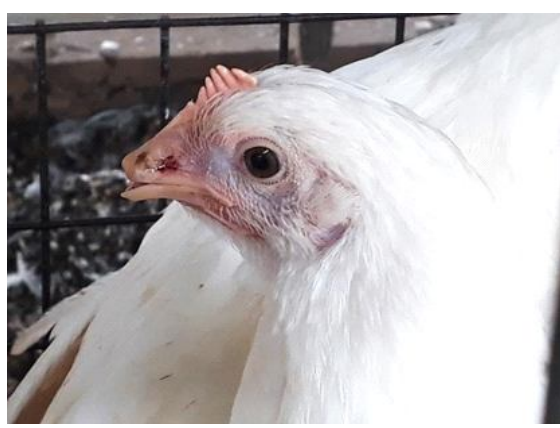

a) Oculonasal discharge with Blood mixed exudate.

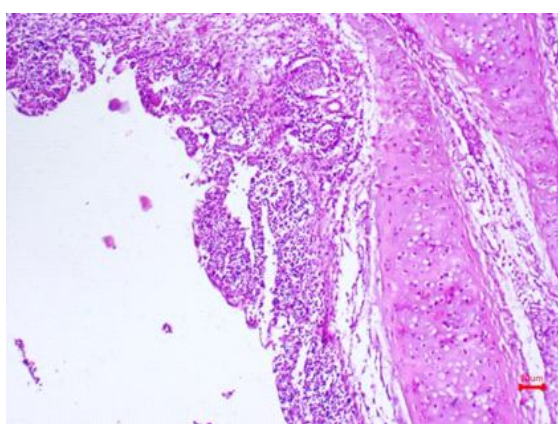

c) Trachea- Haemorrhages with fibrocellular exudate in the

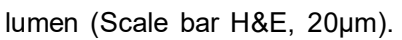

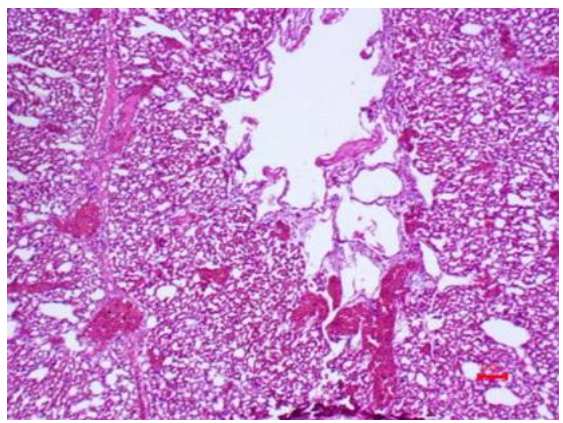

e) Lungs: Congestion and haemorrhagic foci within the parabronchus. (Scale bar H\&E, $10 \mu \mathrm{m}$ ).

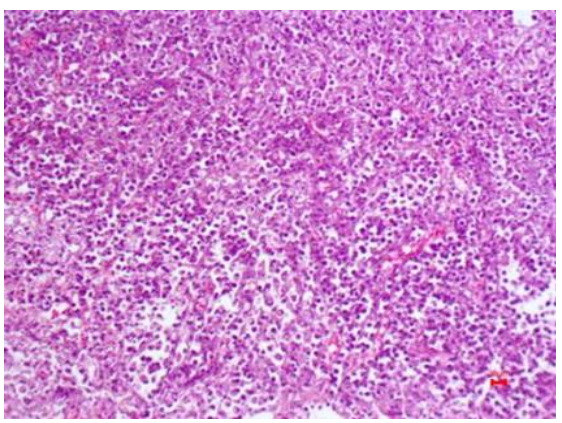

g) Harderian Gland: Mild Lymphoplasmacytic depletion in the acini. (Scale bar H\&E, $10 \mu \mathrm{m}$ ).

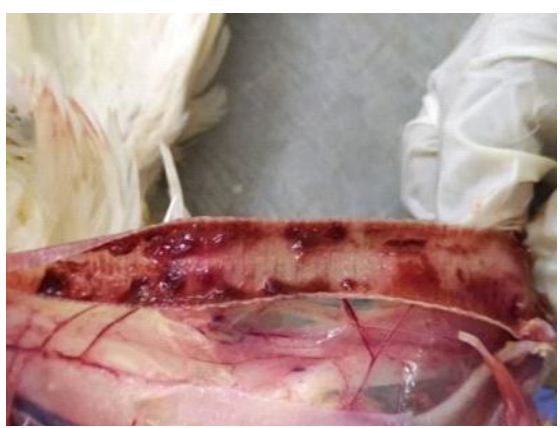

b) Acute Form of Haemorrhagic tracheitis with blood clots in the tracheal lumen.

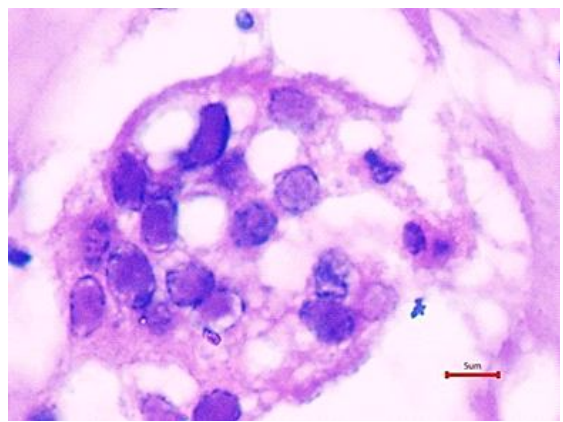

d) Higher magnification of Trachea with formation of multinucleated syncytial cells with eosinophilic intranuclear herpesvirus inclusion bodies in sloughed epithelial cells (Scale bar H\&E, $5 \mu \mathrm{m}$ ).

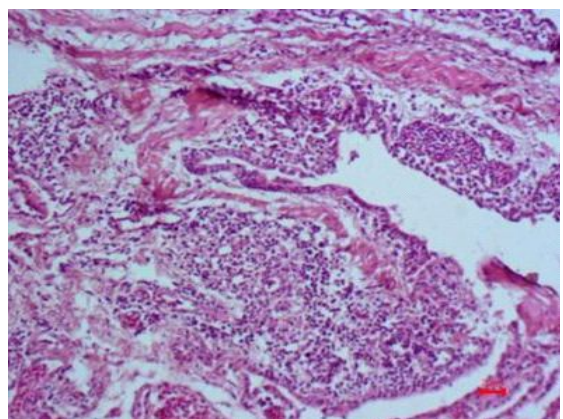

f) Lungs: Submucosal heterophilic infiltration in the Secondary bronchi. (Scale bar H\&E, $10 \mu \mathrm{m}$ ).

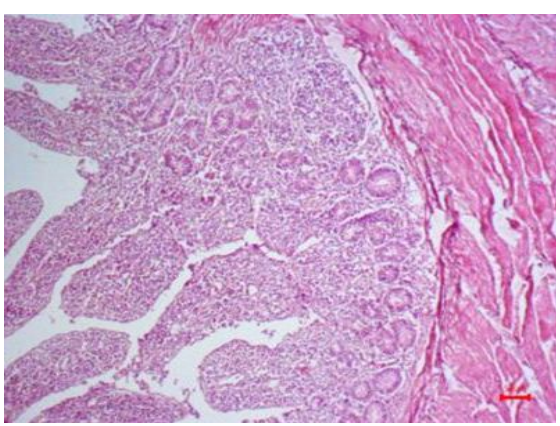

h) Caecal Tonsil: Mild Iymphoid cell depletion in Iymphatic nodule. (Scale bar H\&E, $20 \mu \mathrm{m}$ ).

Fig 1: Different clinico-pathological manifestations of ILTV infection. 


\section{PCR amplification of ICP4 gene}

PCR amplification of extracted DNA from swabs and tissues generated the product size of 635 bp for the GaHV-1 ICP4 gene (Fig 2). The DNA positive control resulted in the amplification of a product with the expected molecular size, while no amplification was observed in the corresponding negative control. The nucleotide sequences obtained aided to compare various virus strains isolated from different geographical areas. Infected cell protein 4 (ICP4) gene is often used to assess the origin of the strain causing disease outbreaks and to differentiate vaccinal or field strain of the virus (Chacón and Ferreira, 2009).

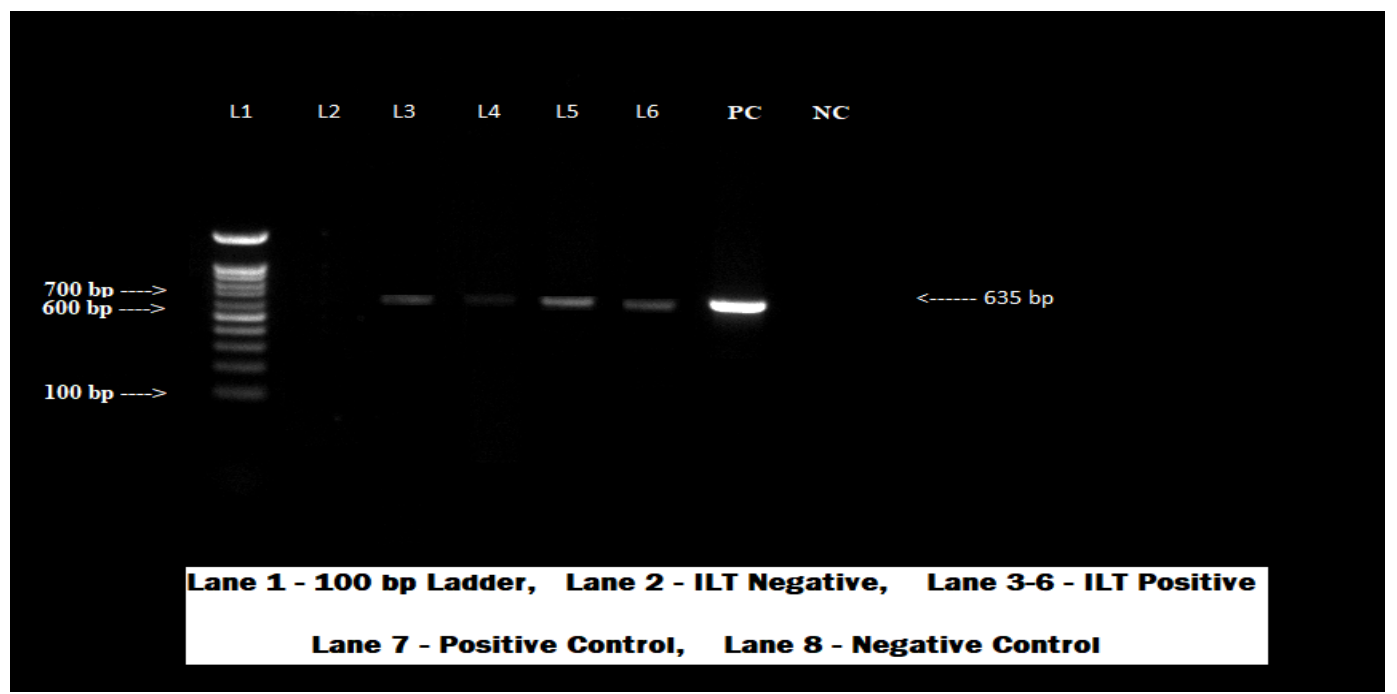

Fig 2: PCR amplification of ICP4 gene from ILTV suspected samples.

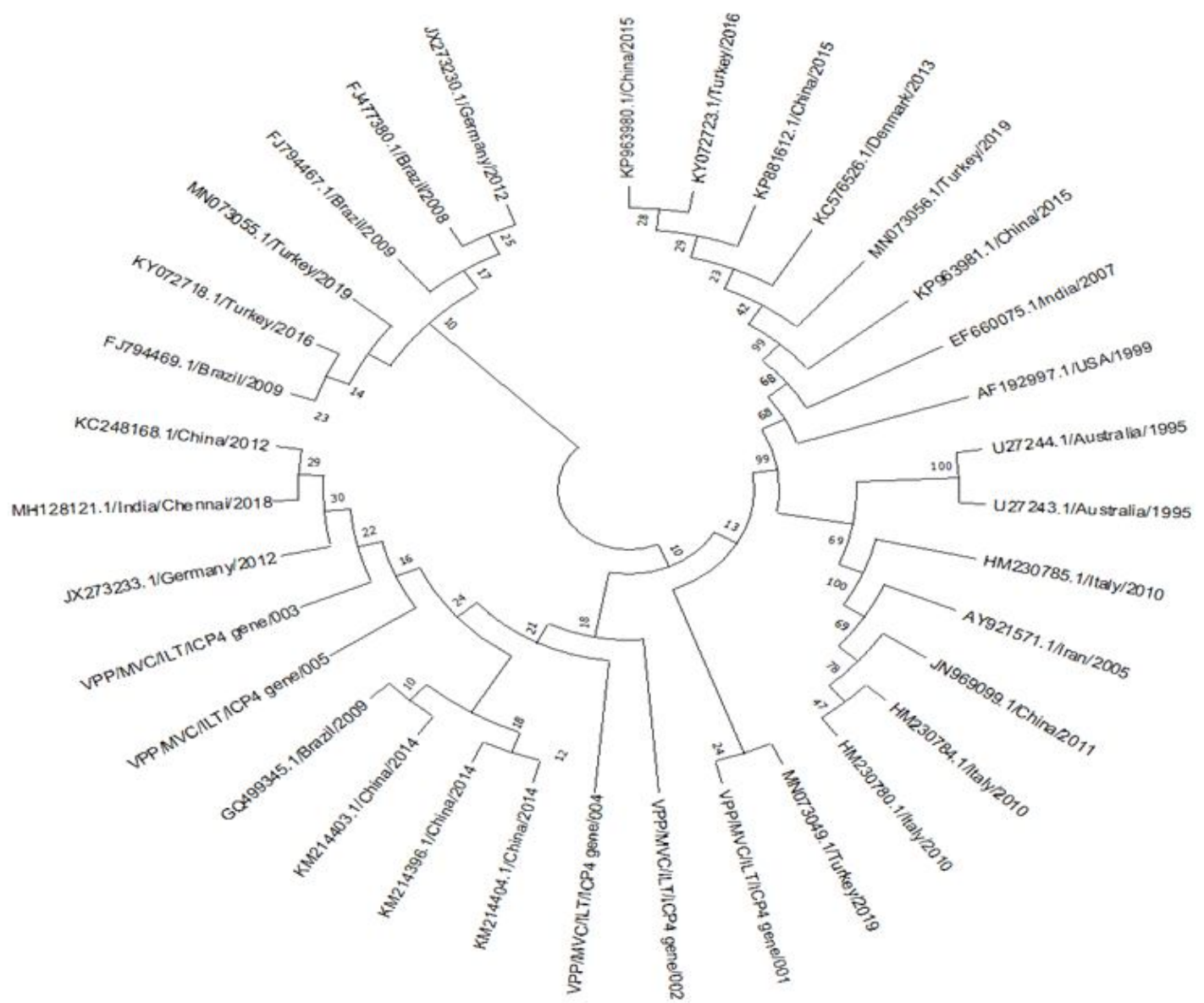

Fig 3: Phylogenetic tree constructed by maximum likelihood method Tamura-Nei model with 1000 bootstrap values. 


\section{Partial genome sequencing and phylogenetic analysis}

Five samples that were found to be positive by PCR were selected for the partial nucleotide sequencing and alignment study for the ICP4 gene and tested for their identity per cent with reference strains obtained via GenBank. The nucleotide sequences obtained from field isolates in this study are available in GenBank database under accession numbers of MZ820427 (VPP/MVC/ILT//CP4gene/001), OK631538 (VPP/MVC/ILT/ICP4gene/002), OK631539 (VPP/MVC/ILT/ ICP4gene/003), OK631540 (VPP/MVC/ILT//CP4gene/004) and OK631541 (VPP/MVC/ILT/ICP4gene/005). The study isolates were $100 \%$ identical to each other but were found to have polymorphic sites when compared to other sequences from India and other countries deposited in GenBank. The phylogenetic analysis based on partial genome sequencing of ICP 4 gene revealed that five isolates of this study were grouped together in a separate branch, apart from other Indian strains, but clustered with the isolates from Turkey, Germany, China and Brazil with $98.99 \%$ homology, as shown in Fig 3. Previous studies have shown that sequencing of a specific region of the genome (ICP4) of GaHV-1 can generate reliable results for differentiation between vaccine and field strains (Chacon and Ferreira, 2009; Gowthaman et al., 2020). A few episodes of clinical disease were still detected in unvaccinated flocks on some farms in the endemic area, indicating that the virus circulating among flocks could produce new outbreaks in unvaccinated chickens.

\section{CONCLUSION}

The molecular characterization and differentiation of GaHV-1 isolates are required for epidemiological studies that aim to elucidate the origin of the virus involved in the outbreak. The present study, along with pathological findings and a rapid conventional $P C R$ assay revealed the highest prevalence of the Infectious laryngotracheitis in layer birds and highly susceptible age group between 20-30 weeks old. Phylogenetic analysis of ICP4 gene revealed high genetic homology between field isolates from commercial layer and broiler flock and the study isolate were clustered with the isolates from Turkey, Germany, China and Brazil. As a result of establishing the pathogenesis of variant Indian ILTV isolate in the present study, will enable in conceiving appropriate diagnosis, vaccination strategy and selecting vaccine type in near future.

\section{ACKNOWLEDGEMENT}

Authors are highly acknowledged to Dean, Madras Veterinary College, Chennai, Dean, Veterinary College and Research Institute, Namakkal and Director, Centre for Animal Production Studies, TANUVAS for providing necessary facilities for the research work.

\section{REFERENCES}

Bagust, T.J., Jones, R.C., Guy, J.S. (2000). Avian infectious laryngotracheitis. Revue scientifique et technique. 19: 483-492.
Bancroft, J.D. and Gamble, M. (2008). Theory and Practice of Histological Techniques. $6^{\text {th }}$ ed. Churchill Livingstone, Elsevier Health Sciences: 1-725.

Blakey, J., Stoute, S., Crossley, B., Mete, A. (2019). Retrospective analysis of infectious laryngotracheitis in backyard chicken flocks in California, 2007-2017 and determination of strain origin by partial ICP4 sequencing. Journal of Veterinary Diagnostic Investigation. 31: 350-358.

Bhutia, L.B. and Singh, D.Y. (2017). Occurrence of Infectious Laryngotracheitis in Poultry Population of Mizoram, India. International Journal of Current Research. 9(06): 51706 $-51710$.

Chacón, J.L. and Ferreira, A.J. (2009). Differentiation of field isolates and vaccine strains of infectious laryngotracheitis virus by DNA sequencing. Vaccine. 27(48): 6731-6738.

Gowthaman, V., Singh, S. D., Dhama, K., Barathidasan, R., Mathapati, B.S., Srinivasan, P., Saravanan, S., Ramakrishnan, M.A. (2014). Molecular detection and characterization of infectious laryngotracheitis virus (Gallid herpesvirus-1) from clinical samples of commercial poultry flocks in India. Virus Disease. 25(3): 345-349.

Gowthaman, V., Kumar, S., Koul, M., Dave, U., Murthy, T., Munuswamy, P., Tiwari, R., Karthik, K., Dhama, K., Michalak, I., Joshi, S.K. (2020). Infectious laryngotracheitis: Etiology, epidemiology, pathobiology and advances in diagnosis and control - a comprehensive review. The Veterinary Quarterly. 40(1): 140-161.

Guy, J.S. and Bagust T.J. (2003) Laryngotracheitis. In: [Saif, Y.M., Barnes, H.J., Glisson, J.R., Fadly, A.M., Dougland, M.C., Swayne, D.E. (eds)] Disease of poultry. $11^{\text {th }}$ edn. lowa State University Press, Ames. 121-134.

Hall, T.A. (1999). BioEdit: a user-friendly biological sequence alignment editor and analysis program for Windows 95/ 98/NT. In: Nucleic Acids Symposium Series. 41: 95-98.

King, A.M.Q., Adams, M.J., Carstens, E.B., Lefkowitz. E.J. (2012). Ninth Report of the International Committee on Taxonomy of Viruses. Elsevier Academic Press, San Diego: 1344.

Kumar, S., Stecher, G., Li, M., Knyaz, C., Tamura, K. (2018). MEGA $\mathrm{X}$ : Molecular Evolutionary Genetics Analysis across computing platforms. Molecular Biology and Eolution. 35: 1547-1549.

Lee, S.W., Devlin, J.M., Markham, J.F., Noormohammadi, A.H., Browning, G.F., Ficorilli, N.P., Hartley, C.A., Markham, P.F. (2013). Phylogenetic and molecular epidemiological studies reveal evidence of multiple past recombination events between infectious laryngotracheitis viruses. PloSone. 8(2). DOI: 10. 1371/journal.pone.0055121.

May, H.G. and Tittsler, R.P. (1925). Tracheolaryngitis in poultry. Journal of American Veterinary Medical Association. 67: 229-231.

Menendez, K.R., Garcia, M., Spatz, S., Tablante, N.L. (2014). Molecular epidemiology of infectious laryngotracheitis: A review. Avian Pathology. 43: 108-117.

Mishra, A., Thangavelu, A., Roy, P., Tirumurugaan, K.G., Hemalatha, S., Gopalakrishnamurthy, T.R., Kirubaharan, J.J. (2020). Infectious laryngotracheitis in layer birds from Tamil Nadu, India. Indian Journal of Animal Research. 54(11): 14081414. 
Mobini, B. (2012). Histological and histochemical studies on the Harderian gland in native chicken. Veterinary Medicine. 57: 404-409.

Moreno, A., Piccirillo, A., Mondin, A., Morandini, E., Gavazzi, L., Cordioli, P. (2010). Epidemic of infectious laryngotracheitis in Italy: Characterization of virus isolates by PCR-restriction fragment length polymorphism and sequence analysis. Avian Diseases. 54(4): 1172-1177.

OIE. (2021). World Organization for Animal Health. Manual of Diagnostic Tests and Vaccines for Terrestrial Animals. Avian Infectious Laryngotracheitis. Chapter. 3.3.3. 810820.

Ou, S.C. and Giambrone, J.J. (2012). Infectious laryngotracheitis virus in chickens. World Journal of Virology. 1(5): 142-149.
Preis, I.S., Braga, J.F., Couto, R.D., Brasil, B.S., Martins, N.R., Ecco, R. (2013). Outbreak of infectious laryngotracheitis in large multi-age egg layer chicken flocks in Minas Gerais, Brazil. Pesquisa Veterinaria Brasileira. 33: 591-596.

Sivaseelan, S., Rajan, T., Malmarugan, S., Balasubramaniam, G.A., Madheswaran, R. (2014). Tissue tropism and pathobiology of infectious laryngotracheitis virus in natural cases of chickens. Israel Journal of Veterinary Medicine. 69: 197202.

Tamura, K. and Nei, M. (1993). Estimation of the number of nucleotide substitutions in the control region of mitochondrial DNA in humans and chimpanzees. Molecular Biology and Evolution. 10: 512- 526. 